Acta Crystallographica Section E

Structure Reports

Online

ISSN 1600-5368

\section{Aqua[ $N^{\prime}$-(3-ethoxy-2-oxidobenzyl- $\left.\kappa O\right)$ - furan-1-carbohydrazidato- $\left.\kappa^{2} N^{\prime}, O\right]-$ dioxidomolybdenum(VI)-4, ' $^{\prime}$-bipyridine} $(2 / 1)$

\section{Ngui Khiong Ngan, Richard Chee Seng Wong, Kong Mun Lo and Seik Weng Ng*}

Department of Chemistry, University of Malaya, 50603 Kuala Lumpur, Malaysia Correspondence e-mail: seikweng@um.edu.my

Received 5 May 2011; accepted 7 May 2011

Key indicators: single-crystal X-ray study; $T=100 \mathrm{~K}$; mean $\sigma(\mathrm{C}-\mathrm{C})=0.004 \AA$; disorder in main residue; $R$ factor $=0.026 ; w R$ factor $=0.076$; data-to-parameter ratio $=16.2$

The $\mathrm{Mo}^{\mathrm{VI}}$ atom in the title co-crystal, $\left[\mathrm{Mo}\left(\mathrm{C}_{14} \mathrm{H}_{12} \mathrm{~N}_{2} \mathrm{O}_{4}\right) \mathrm{O}_{2^{-}}\right.$ $\left.\left(\mathrm{H}_{2} \mathrm{O}\right)\right] \cdot 0.5 \mathrm{C}_{10} \mathrm{H}_{8} \mathrm{~N}_{2}$, is $O, N, O^{\prime}$-chelated by the deprotonated Schiff base and coordinated by the oxide and water $\mathrm{O}$ atoms in an octahedral geometry. The five-membered chelate ring is planar (r.m.s. deviation $=0.019 \AA$ ), but the six-membered chelate ring is puckered (r.m.s. deviation $=0.108 \AA$ ). Two mononuclear molecules are linked across a center of inversion by an $\mathrm{O}-\mathrm{H}_{\text {water }} \cdot \mathrm{O}$ hydrogen bond; adjacent dinuclear units are linked by an water-4, $4^{\prime}$-bipyridine $\mathrm{O}-\mathrm{H} \cdots \mathrm{N}$ hydrogen bond, generating a linear chain structure. The 4,4'-bipyridine molecule is disordered over two positions in a 1:1 ratio.

\section{Related literature}

For a related $\mathrm{Mo}^{\mathrm{VI}} \mathrm{O}_{2}-4^{\prime}$,4-bipyridine adduct, see: Dinda et al. (2006).

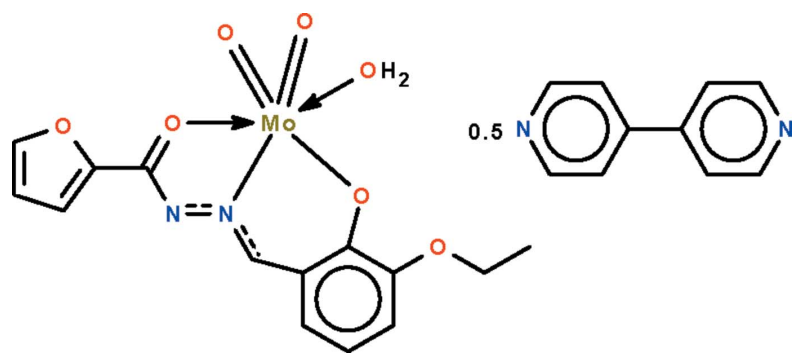

\section{Experimental}

Crystal data

$\left[\mathrm{Mo}\left(\mathrm{C}_{14} \mathrm{H}_{12} \mathrm{~N}_{2} \mathrm{O}_{4}\right) \mathrm{O}_{2}\right.$ -

$\left.\left(\mathrm{H}_{2} \mathrm{O}\right)\right] \cdot 0.5 \mathrm{C}_{10} \mathrm{H}_{8} \mathrm{~N}_{2}$

$M_{r}=496.30$

Triclinic, $P \overline{1}$

$a=7.9237$ (1) §

$b=10.1869$ (1) $\AA$

$c=13.3215(2) \AA$

$\alpha=78.7841(5)^{\circ}$

$\beta=78.4605(5)^{\circ}$

$\gamma=69.5728(5)^{\circ}$

$V=978.15(2) \AA^{3}$

$Z=2$

Mo $K \alpha$ radiation

$\mu=0.72 \mathrm{~mm}^{-1}$

$T=100 \mathrm{~K}$

$0.2 \times 0.2 \times 0.2 \mathrm{~mm}$

\section{Data collection}

Bruker SMART APEX

diffractometer

Absorption correction: multi-scan (SADABS; Sheldrick, 1996)

$T_{\min }=0.649, T_{\max }=0.746$

9175 measured reflections 4445 independent reflections 4266 reflections with $I>2 \sigma(I)$ $R_{\text {int }}=0.019$

Refinement

$R\left[F^{2}>2 \sigma\left(F^{2}\right)\right]=0.026$

$w R\left(F^{2}\right)=0.076$

$S=0.98$

4445 reflections

275 parameters

24 restraints

$\mathrm{H}$ atoms treated by a mixture of independent and constrained refinement

$\Delta \rho_{\max }=0.73{\mathrm{e} \AA^{-3}}^{-3}$

$\Delta \rho_{\min }=-0.72 \mathrm{e}^{-3}$

Table 1

Hydrogen-bond geometry $\left(\AA{ }^{\circ}\right)$.

\begin{tabular}{lllll}
\hline$D-\mathrm{H} \cdots A$ & $D-\mathrm{H}$ & $\mathrm{H} \cdots A$ & $D \cdots A$ & $D-\mathrm{H} \cdots A$ \\
\hline $\mathrm{O} 1 \mathrm{w}-\mathrm{H} 11 \cdots \mathrm{N} 3$ & $0.83(1)$ & $1.86(1)$ & $2.689(3)$ & $174(3)$ \\
$\mathrm{O} 1 \mathrm{w}-\mathrm{H} 12 \cdots \mathrm{N} 1{ }^{\mathrm{i}}$ & $0.84(1)$ & $1.97(1)$ & $2.794(2)$ & $167(3)$ \\
\hline
\end{tabular}

Symmetry code: (i) $-x+2,-y+1,-z$.

Data collection: APEX2 (Bruker, 2009); cell refinement: SAINT (Bruker, 2009); data reduction: $S A I N T$; program(s) used to solve structure: SHELXS97 (Sheldrick, 2008); program(s) used to refine structure: SHELXL97 (Sheldrick, 2008); molecular graphics: $X$ SEED (Barbour, 2001); software used to prepare material for publication: publCIF (Westrip, 2010).

We thank the University of Malaya (grant No. RG020/ 09AFR) for supporting this study.

Supplementary data and figures for this paper are available from the IUCr electronic archives (Reference: JH2290).

\title{
References
}

Barbour, L. J. (2001). J. Supramol. Chem. 1, 189-191.

Bruker (2009). APEX2 and SAINT. Bruker AXS Inc., Madison, Wisconsin, USA.

Dinda, R., Ghosh, S., Falvello, L. R., Tomas, M. \& Mak, T. C. W. (2006). Polyhedron, 25, 2375-2382.

Sheldrick, G. M. (1996). SADABS. University of Göttingen, Germany.

Sheldrick, G. M. (2008). Acta Cryst. A64, 112-122.

Westrip, S. P. (2010). J. Appl. Cryst. 43, 920-925. 


\section{supporting information}

Acta Cryst. (2011). E67, m748 [doi:10.1107/S1600536811017260]

\section{Aqua[N'-(3-ethoxy-2-oxidobenzyl- $\kappa O)$ furan-1-carbohydrazidato- $\left.\kappa^{2} N^{\prime}, O\right]$ dioxidomolybdenum(VI)-4,4'-bipyridine (2/1)}

\section{Ngui Khiong Ngan, Richard Chee Seng Wong, Kong Mun Lo and Seik Weng Ng}

\section{S1. Comment}

The Schiff bases that are synthesized by condensing salicylaldehyde (and its substituted analogs) with aroylhydrazides (and their substituted analogs) function as terdentate $O, N, O^{\prime}$-chelates to a wide range of metal ions. A large number of metal derivatives have been reported; in octahedral systems, the ligand generally exists as a doubly-deprotonated species that chelates in a fac manner. A dioxomolybdenum(VI) derivative is known in which 4,4'-bipyridine binds to two metal atoms (Dinda et al., 2006). In the present study, a furan-type of Schiff base leads to a water-coordinated derivative in which 4,4'-bipyridine interacts indirectly, through the water molecule, in an outer-sphere coordination mode. The $\mathrm{Mo}^{\mathrm{VI}}$ atom in the co-crystal, $\mathrm{MoO}_{2}\left(\mathrm{H}_{2} \mathrm{O}\right)\left(\mathrm{C}_{14} \mathrm{H}_{12} \mathrm{~N}_{2} \mathrm{O}_{4}\right) \cdot 0.5 \mathrm{C}_{10} \mathrm{H}_{10} \mathrm{~N}_{2}$, is $O, N, O^{\prime}$-chelated by the deprotonated Schiff base and coordinated by the oxo and water $\mathrm{O}$ atoms in an octahedral geometry (Scheme I, Fig. 1). The five-membed chelate ring is planar [r.m.s. deviation $0.019 \AA$ ] but the six-membered chelate ring is puckered [r.m.s. deviation $0.108 \AA$ ]. Two mononuclear molecules are linked across a center-of-inversion by an $\mathrm{O}-\mathrm{H}_{\text {water }} \cdots \mathrm{O}$ hydrogen bond; adjacent dinuclear units are linked by an $O-\mathrm{H}_{\text {water }} \cdots \mathrm{N}_{4,4^{\prime}-\text {-bipyridine }}$ hydrogen bond to generate a linear chain structure (Table 1). The 4,4'-bipyridine molecule is disordered over two positions in a 1:1 ratio.

\section{S2. Experimental}

3-Ethoxysalicylaldehyde ( $0.166 \mathrm{~g}, 1 \mathrm{mmol})$ and 2-furoylhydrazide $(0.120 \mathrm{~g}, 1 \mathrm{mmol})$ were condensed in methanol (100 $\mathrm{ml})$. The solution was heated to give a yellow coloration. The cool solution yielded the desired Schiff base as a yellow compound. The ligand $(0.270 \mathrm{~g}, 1 \mathrm{mmol})$ and di(acetylacetonato)dioxomolybdenum(VI) $(0.328 \mathrm{~g}, 1 \mathrm{mmol})$ were dissolved in heated in methanol for an hour. To the orange solution was added 4,4'-bipyridine ( $0.08 \mathrm{~g}, 0.5 \mathrm{mmol})$; heating was continued for another hour. The solution was filtered and set aside for the growth of crystals, m.p. 495-497 K.

\section{S3. Refinement}

Carbon-bound H-atoms were placed in calculated positions ( $\mathrm{C}-\mathrm{H} 0.95$ to $0.99 \AA)$ and were included in the refinement in the riding model approximation, with $U(\mathrm{H})$ set to 1.2 times $U_{\text {eq }}(\mathrm{C})$.

The water $\mathrm{H}$-atoms were located in a difference Fourier map and were refined with distance restraints of $\mathrm{O}-\mathrm{H}$ $0.84 \pm 0.01$ and $\mathrm{H} \cdots \mathrm{H} 1.37 \pm 0.01 \AA$; their temperature factors were refined.

The 4,4'-bipyridine molecule is disordered about a center-of-inversion. The pyridyl ring was refined as two rings that shared common $\mathrm{N}$ and and $\mathrm{C}_{\text {para }}$ atoms. As the occupancy refined to nearly $1 / 2$, the occupancy was then fixed as 0.5 . Carbon-nitrogen distances were restrained to $1.35 \pm 0.01 \AA$ and carbon-carbon distances to $1.39 \pm 0.01 \AA$. The six atoms of each ring were restrained to lie on a plane. Attempts to refined the disordered atoms anisotropically led to non-positive definites; the eight disordered atoms were then refined only isotropically. 
Omitted from the refinement owing to bad disagreement were these reflections: $\left(\begin{array}{lll}0 & 0 & 1\end{array}\right),(-6$ - 6 2), (4 - 5 4) and (3 9 7).

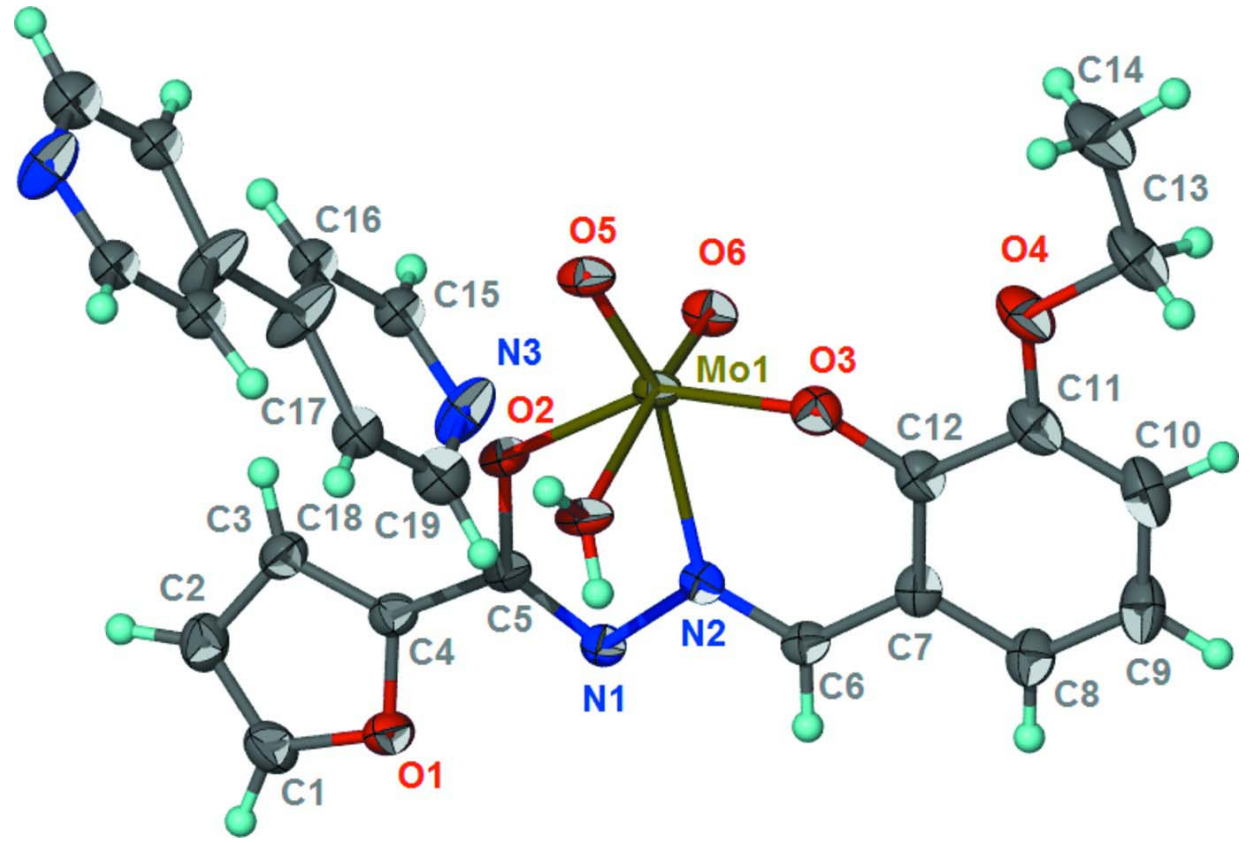

\section{Figure 1}

Thermal ellipsoid plot (Barbour, 2001) of $\mathrm{MoO}_{2}\left(\mathrm{H}_{2} \mathrm{O}\right)\left(\mathrm{C}_{14} \mathrm{H}_{12} \mathrm{~N}_{2} \mathrm{O}_{4}\right) \cdot 0.5 \mathrm{C}_{10} \mathrm{H}_{10} \mathrm{~N}_{2}$ at the $70 \%$ probability level; hydrogen atoms are drawn as spheres of arbitrary radius. The disorder in the 4,4'-bipyridine molecule is not shown.

Aqua[ $N^{\prime}$-(3-ethoxy-2-oxidobenzyl- $\left.\kappa O\right)$ furan-1-carbohydrazidato- $\left.\kappa^{2} N^{\prime}, O\right]$ dioxidomolybdenum(VI)-4,4'bipyridine (2/1)

Crystal data

$\left[\mathrm{Mo}\left(\mathrm{C}_{14} \mathrm{H}_{12} \mathrm{~N}_{2} \mathrm{O}_{4}\right) \mathrm{O}_{2}\left(\mathrm{H}_{2} \mathrm{O}\right)\right] \cdot 0.5 \mathrm{C}_{10} \mathrm{H}_{8} \mathrm{~N}_{2}$ $Z=2$

$M_{r}=496.30$

Triclinic, $P \overline{1}$

Hall symbol: -P 1

$a=7.9237(1) \AA$

$b=10.1869(1) \AA$

$c=13.3215(2) \AA$

$\alpha=78.7841(5)^{\circ}$

$\beta=78.4605(5)^{\circ}$

$\gamma=69.5728(5)^{\circ}$

$V=978.15(2) \AA^{3}$

$$
F(000)=502
$$

$D_{\mathrm{x}}=1.685 \mathrm{Mg} \mathrm{m}^{-3}$

Mo $K \alpha$ radiation, $\lambda=0.71073 \AA$

Cell parameters from 8273 reflections

$\theta=2.5-28.2^{\circ}$

$\mu=0.72 \mathrm{~mm}^{-1}$

$T=100 \mathrm{~K}$

Block, orange

$0.2 \times 0.2 \times 0.2 \mathrm{~mm}$

\section{Data collection}

\section{Bruker SMART APEX}

diffractometer

Radiation source: fine-focus sealed tube

Graphite monochromator

$\omega$ scans

Absorption correction: multi-scan

(SADABS; Sheldrick, 1996)

$T_{\min }=0.649, T_{\max }=0.746$

9175 measured reflections

4445 independent reflections

4266 reflections with $I>2 \sigma(I)$

$R_{\text {int }}=0.019$

$\theta_{\max }=27.5^{\circ}, \theta_{\min }=2.2^{\circ}$

$h=-10 \rightarrow 10$

$k=-13 \rightarrow 13$

$l=-17 \rightarrow 17$ 


\section{Refinement}

Refinement on $F^{2}$

Least-squares matrix: full

$R\left[F^{2}>2 \sigma\left(F^{2}\right)\right]=0.026$

$w R\left(F^{2}\right)=0.076$

$S=0.98$

4445 reflections

275 parameters

24 restraints

Primary atom site location: structure-invariant direct methods
Secondary atom site location: difference Fourier map

Hydrogen site location: inferred from neighbouring sites

$\mathrm{H}$ atoms treated by a mixture of independent and constrained refinement

$w=1 /\left[\sigma^{2}\left(F_{\mathrm{o}}{ }^{2}\right)+(0.0474 P)^{2}+1.0897 P\right]$ where $P=\left(F_{\mathrm{o}}^{2}+2 F_{\mathrm{c}}{ }^{2}\right) / 3$

$(\Delta / \sigma)_{\max }=0.001$

$\Delta \rho_{\max }=0.73$ e $\AA^{-3}$

$\Delta \rho_{\min }=-0.72$ e $\AA^{-3}$

Fractional atomic coordinates and isotropic or equivalent isotropic displacement parameters $\left(\AA^{2}\right)$

\begin{tabular}{|c|c|c|c|c|c|}
\hline & $x$ & $y$ & $z$ & $U_{\text {iso }} * / U_{\text {eq }}$ & Occ. $(<1)$ \\
\hline Mo1 & $0.74598(2)$ & $0.304217(17)$ & $0.170071(12)$ & $0.01695(7)$ & \\
\hline $\mathrm{O} 1$ & $1.1355(2)$ & $0.30269(16)$ & $-0.20486(13)$ & $0.0246(3)$ & \\
\hline $\mathrm{O} 2$ & $0.9252(2)$ & $0.21432(15)$ & $0.05147(12)$ & $0.0195(3)$ & \\
\hline $\mathrm{O} 3$ & $0.6036(2)$ & $0.46573(17)$ & $0.23935(12)$ & 0.0218 & \\
\hline $\mathrm{O} 4$ & $0.3337(2)$ & $0.5786(2)$ & $0.37565(13)$ & $0.0304(4)$ & \\
\hline O5 & $0.8232(2)$ & $0.17630(17)$ & $0.26946(12)$ & $0.0224(3)$ & \\
\hline $\mathrm{O} 6$ & $0.5697(2)$ & $0.26804(17)$ & $0.13977(12)$ & 0.0223 & \\
\hline $\mathrm{O} 1 \mathrm{~W}$ & $0.9855(2)$ & $0.36664(17)$ & $0.18299(12)$ & $0.0210(3)$ & \\
\hline H11 & $1.047(3)$ & $0.327(3)$ & $0.2310(16)$ & $0.028(8)^{*}$ & \\
\hline H12 & $1.017(4)$ & $0.435(2)$ & $0.150(2)$ & $0.043(9)^{*}$ & \\
\hline N1 & $0.8802(2)$ & $0.43227(18)$ & $-0.05053(13)$ & $0.0173(3)$ & \\
\hline N2 & $0.7547(2)$ & $0.47579(18)$ & $0.03625(13)$ & $0.0163(3)$ & \\
\hline N3 & $1.1836(3)$ & $0.2227(2)$ & $0.33511(17)$ & $0.0305(5)$ & \\
\hline $\mathrm{C} 1$ & $1.2652(3)$ & $0.2079(3)$ & -0.26360 & $0.0270(5)$ & \\
\hline $\mathrm{H} 1$ & 1.3224 & 0.2325 & -0.3310 & $0.032 *$ & \\
\hline $\mathrm{C} 2$ & $1.3007(3)$ & $0.0757(3)$ & $-0.2131(2)$ & $0.0275(5)$ & \\
\hline $\mathrm{H} 2$ & 1.3847 & -0.0080 & -0.2377 & $0.033^{*}$ & \\
\hline $\mathrm{C} 3$ & $1.1882(3)$ & $0.0854(2)$ & $-0.11569(18)$ & $0.0229(4)$ & \\
\hline $\mathrm{H} 3$ & 1.1818 & 0.0099 & -0.0622 & $0.027 *$ & \\
\hline $\mathrm{C} 4$ & $1.0916(3)$ & $0.2245(2)$ & $-0.11444(16)$ & $0.0193(4)$ & \\
\hline $\mathrm{C} 5$ & 0.9575 & $0.2954(2)$ & $-0.03452(16)$ & $0.0176(4)$ & \\
\hline C6 & $0.6621(3)$ & $0.6087(2)$ & $0.02969(16)$ & $0.0179(4)$ & \\
\hline H6 & 0.6873 & 0.6688 & -0.0314 & $0.022 *$ & \\
\hline $\mathrm{C} 7$ & 0.5219 & $0.6710(2)$ & $0.11060(16)$ & $0.0197(4)$ & \\
\hline $\mathrm{C} 8$ & 0.4088 & $0.8115(2)$ & $0.08794(18)$ & $0.0240(4)$ & \\
\hline H8 & 0.4312 & 0.8640 & 0.0224 & $0.029 *$ & \\
\hline C9 & $0.2662(3)$ & $0.8737(3)$ & $0.1600(2)$ & $0.0297(5)$ & \\
\hline H9 & 0.1900 & 0.9680 & 0.1436 & $0.036^{*}$ & \\
\hline $\mathrm{C} 10$ & 0.2339 & 0.7978 & $0.25712(19)$ & $0.0304(5)$ & \\
\hline $\mathrm{H} 10$ & 0.1334 & 0.8398 & 0.3059 & $0.036^{*}$ & \\
\hline C11 & $0.3482(3)$ & $0.6612(3)$ & $0.28256(18)$ & $0.0252(5)$ & \\
\hline $\mathrm{C} 12$ & $0.4943(3)$ & $0.5964(2)$ & $0.20924(16)$ & $0.0207(4)$ & \\
\hline C13 & $0.1903(3)$ & $0.6364(3)$ & $0.45495(19)$ & $0.0340(6)$ & \\
\hline
\end{tabular}




\begin{tabular}{|c|c|c|c|c|c|}
\hline H13A & 0.0704 & 0.6616 & 0.4318 & $0.041^{*}$ & \\
\hline H13B & 0.2028 & 0.7225 & 0.4722 & $0.041^{*}$ & \\
\hline C14 & $0.2070(4)$ & $0.5235(4)$ & $0.5478(2)$ & $0.0386(6)$ & \\
\hline H14A & 0.1101 & 0.5580 & 0.6041 & $0.058^{*}$ & \\
\hline H14B & 0.3257 & 0.5005 & 0.5703 & $0.058^{*}$ & \\
\hline $\mathrm{H} 14 \mathrm{C}$ & 0.1960 & 0.4385 & 0.5293 & $0.058^{*}$ & \\
\hline C15 & $1.2028(5)$ & 0.0903 (4) & 0.3618 & $0.0214(8)^{*}$ & 0.50 \\
\hline H15 & 1.1241 & 0.0543 & 0.3384 & $0.026^{*}$ & 0.50 \\
\hline $\mathrm{C} 16$ & $1.3288(5)$ & -0.0028 & $0.4216(3)$ & $0.0206(8)^{*}$ & 0.50 \\
\hline H16 & 1.3433 & -0.1009 & 0.4325 & $0.025^{*}$ & 0.50 \\
\hline $\mathrm{C} 15^{\prime}$ & $1.1327(6)$ & $0.1138(4)$ & $0.4102(3)$ & $0.0199(8)^{*}$ & 0.50 \\
\hline H15' & 1.0185 & 0.1015 & 0.4124 & $0.024 *$ & 0.50 \\
\hline $\mathrm{C} 16^{\prime}$ & $1.2513(5)$ & $0.0266(4)$ & $0.4794(3)$ & $0.0208(8)^{*}$ & 0.50 \\
\hline H16' & 1.2182 & -0.0413 & 0.5320 & $0.025^{*}$ & 0.50 \\
\hline $\mathrm{C} 17$ & $1.4335(4)$ & $0.0471(2)$ & $0.4655(2)$ & $0.0340(6)$ & \\
\hline C18 & $1.4347(8)$ & $0.1850(6)$ & $0.4256(5)$ & $0.0223(19)^{*}$ & 0.50 \\
\hline H18 & 1.5229 & 0.2190 & 0.4409 & $0.027 *$ & 0.50 \\
\hline C19 & $1.3061(8)$ & $0.2709(7)$ & $0.3637(4)$ & $0.0284(19)^{*}$ & 0.50 \\
\hline H19 & 1.3025 & 0.3660 & 0.3403 & $0.034 *$ & 0.50 \\
\hline C18' & $1.4505(8)$ & $0.1689(6)$ & $0.4113(5)$ & $0.0148(14)^{*}$ & 0.50 \\
\hline H18' & 1.5499 & 0.1977 & 0.4166 & $0.018 *$ & 0.50 \\
\hline C19' & $1.3228(6)$ & $0.2528(5)$ & $0.3477(4)$ & $0.0116(11)^{*}$ & 0.50 \\
\hline H19' & 1.3389 & 0.3383 & 0.3109 & $0.014 *$ & 0.50 \\
\hline
\end{tabular}

Atomic displacement parameters $\left(\AA^{2}\right)$

\begin{tabular}{lllllll}
\hline & $U^{11}$ & $U^{22}$ & $U^{33}$ & $U^{12}$ & $U^{13}$ & $U^{23}$ \\
\hline Mo1 & $0.01730(11)$ & $0.01891(11)$ & $0.01575(11)$ & $-0.00872(7)$ & $-0.00505(7)$ & $0.00321(7)$ \\
O1 & $0.0285(8)$ & $0.0188(7)$ & $0.0251(8)$ & $-0.0096(6)$ & $0.0024(6)$ & $-0.0023(6)$ \\
O2 & $0.0230(7)$ & $0.0158(7)$ & $0.0197(7)$ & $-0.0081(6)$ & $-0.0036(6)$ & $0.0017(5)$ \\
O3 & $0.0227(7)$ & $0.0251(8)$ & $0.0170(7)$ & $-0.0075(6)$ & $-0.0037(6)$ & $-0.0008(6)$ \\
O4 & $0.0255(8)$ & $0.0428(10)$ & $0.0201(8)$ & $-0.0114(8)$ & $0.0031(6)$ & $-0.0039(7)$ \\
O5 & $0.0226(7)$ & $0.0246(8)$ & $0.0220(7)$ & $-0.0124(6)$ & $-0.0085(6)$ & $0.0068(6)$ \\
O6 & $0.0212(7)$ & $0.0279(8)$ & $0.0204(7)$ & $-0.0117(6)$ & $-0.0057(6)$ & $0.0007(6)$ \\
O1W & $0.0224(8)$ & $0.0227(8)$ & $0.0212(7)$ & $-0.0133(6)$ & $-0.0097(6)$ & $0.0074(6)$ \\
N1 & $0.0178(8)$ & $0.0179(8)$ & $0.0163(8)$ & $-0.0066(7)$ & $-0.0023(6)$ & $-0.0013(6)$ \\
N2 & $0.0162(8)$ & $0.0193(8)$ & $0.0140(8)$ & $-0.0062(7)$ & $-0.0041(6)$ & $-0.0011(6)$ \\
N3 & $0.0383(12)$ & $0.0186(9)$ & $0.0381(12)$ & $-0.0039(8)$ & $-0.0241(9)$ & $-0.0027(8)$ \\
C1 & $0.0269(11)$ & $0.0288(11)$ & $0.0255(11)$ & $-0.0111(9)$ & $0.0035(9)$ & $-0.0081(9)$ \\
C2 & $0.0260(11)$ & $0.0220(11)$ & $0.0355(13)$ & $-0.0066(9)$ & $-0.0012(9)$ & $-0.0116(9)$ \\
C3 & $0.0216(10)$ & $0.0179(10)$ & $0.0285(11)$ & $-0.0063(8)$ & $-0.0049(8)$ & $-0.0006(8)$ \\
C4 & $0.0196(9)$ & $0.0186(10)$ & $0.0217(10)$ & $-0.0087(8)$ & $-0.0045(8)$ & $-0.0010(8)$ \\
C5 & $0.0177(9)$ & $0.0182(9)$ & $0.0192(9)$ & $-0.0082(8)$ & $-0.0062(7)$ & $0.0002(7)$ \\
C6 & $0.0191(9)$ & $0.0193(9)$ & $0.0165(9)$ & $-0.0071(8)$ & $-0.0065(7)$ & $0.0006(7)$ \\
C7 & $0.0183(9)$ & $0.0222(10)$ & $0.0198(10)$ & $-0.0054(8)$ & $-0.0063(8)$ & $-0.0046(8)$ \\
C8 & $0.0235(11)$ & $0.0244(11)$ & $0.0223(10)$ & $-0.0030(9)$ & $-0.0078(8)$ & $-0.0036(8)$ \\
C9 & $0.0230(11)$ & $0.0308(12)$ & $0.0308(12)$ & $0.0022(9)$ & $-0.0088(9)$ & $-0.0091(10)$ \\
C10 & $0.0195(10)$ & $0.0425(14)$ & $0.0272(12)$ & $-0.0035(10)$ & $-0.0025(9)$ & $-0.0133(10)$
\end{tabular}




\begin{tabular}{lllllll}
$\mathrm{C} 11$ & $0.0204(10)$ & $0.0371(13)$ & $0.0209(10)$ & $-0.0115(9)$ & $-0.0031(8)$ & $-0.0061(9)$ \\
$\mathrm{C} 12$ & $0.0185(10)$ & $0.0252(10)$ & $0.0203(10)$ & $-0.0077(8)$ & $-0.0053(8)$ & $-0.0039(8)$ \\
$\mathrm{C} 13$ & $0.0256(12)$ & $0.0534(16)$ & $0.0240(11)$ & $-0.0151(11)$ & $0.0054(9)$ & $-0.0122(11)$ \\
C14 & $0.0331(14)$ & $0.0623(19)$ & $0.0219(12)$ & $-0.0197(13)$ & $0.0026(10)$ & $-0.0080(12)$ \\
C17 & $0.0435(15)$ & $0.0168(10)$ & $0.0499(16)$ & $-0.0094(10)$ & $-0.0341(13)$ & $0.0040(10)$ \\
\hline
\end{tabular}

Geometric parameters $\left(\hat{A},{ }^{\circ}\right)$

\begin{tabular}{|c|c|c|c|}
\hline Mo1-O6 & $1.7007(15)$ & $\mathrm{C} 7-\mathrm{C} 8$ & $1.410(3)$ \\
\hline Mo1-O5 & $1.7093(15)$ & $\mathrm{C} 8-\mathrm{C} 9$ & $1.379(3)$ \\
\hline Mo1-O3 & $1.9262(16)$ & $\mathrm{C} 8-\mathrm{H} 8$ & 0.9500 \\
\hline $\mathrm{Mo} 1-\mathrm{O} 2$ & $2.0270(15)$ & $\mathrm{C} 9-\mathrm{C} 10$ & $1.398(4)$ \\
\hline Mo1-O1W & $2.2479(15)$ & C9- $\mathrm{H} 9$ & 0.9500 \\
\hline Mo1-N2 & $2.2495(17)$ & $\mathrm{C} 10-\mathrm{C} 11$ & $1.389(4)$ \\
\hline $\mathrm{O} 1-\mathrm{C} 4$ & $1.364(3)$ & $\mathrm{C} 10-\mathrm{H} 10$ & 0.9500 \\
\hline $\mathrm{O} 1-\mathrm{C} 1$ & $1.371(3)$ & $\mathrm{C} 11-\mathrm{C} 12$ & $1.413(3)$ \\
\hline $\mathrm{O} 2-\mathrm{C} 5$ & $1.313(2)$ & $\mathrm{C} 13-\mathrm{C} 14$ & 1.509 (4) \\
\hline $\mathrm{O} 3-\mathrm{C} 12$ & $1.345(3)$ & $\mathrm{C} 13-\mathrm{H} 13 \mathrm{~A}$ & 0.9900 \\
\hline $\mathrm{O} 4-\mathrm{C} 11$ & $1.365(3)$ & $\mathrm{C} 13-\mathrm{H} 13 \mathrm{~B}$ & 0.9900 \\
\hline $\mathrm{O} 4-\mathrm{C} 13$ & $1.431(3)$ & $\mathrm{C} 14-\mathrm{H} 14 \mathrm{~A}$ & 0.9800 \\
\hline $\mathrm{O} 1 \mathrm{~W}-\mathrm{H} 11$ & $0.833(10)$ & $\mathrm{C} 14-\mathrm{H} 14 \mathrm{~B}$ & 0.9800 \\
\hline $\mathrm{O} 1 \mathrm{~W}-\mathrm{H} 12$ & $0.837(11)$ & $\mathrm{C} 14-\mathrm{H} 14 \mathrm{C}$ & 0.9800 \\
\hline $\mathrm{N} 1-\mathrm{C} 5$ & $1.305(3)$ & $\mathrm{C} 15-\mathrm{C} 16$ & $1.377(5)$ \\
\hline $\mathrm{N} 1-\mathrm{N} 2$ & $1.398(2)$ & $\mathrm{C} 15-\mathrm{H} 15$ & 0.9500 \\
\hline $\mathrm{N} 2-\mathrm{C} 6$ & $1.291(3)$ & $\mathrm{C} 16-\mathrm{C} 17$ & $1.376(4)$ \\
\hline N3-C15 & $1.288(4)$ & $\mathrm{C} 16-\mathrm{H} 16$ & 0.9500 \\
\hline N3-C19' & $1.291(5)$ & $\mathrm{C} 15^{\prime}-\mathrm{C} 16^{\prime}$ & $1.403(5)$ \\
\hline N3-C19 & $1.371(6)$ & $\mathrm{C} 15^{\prime}-\mathrm{H} 15^{\prime}$ & 0.9500 \\
\hline $\mathrm{N} 3-\mathrm{C} 15^{\prime}$ & $1.454(4)$ & $\mathrm{C} 16^{\prime}-\mathrm{C} 17$ & $1.500(5)$ \\
\hline $\mathrm{C} 1-\mathrm{C} 2$ & $1.344(4)$ & $\mathrm{C} 16^{\prime}-\mathrm{H} 16^{\prime}$ & 0.9500 \\
\hline $\mathrm{C} 1-\mathrm{H} 1$ & 0.9500 & $\mathrm{C} 17-\mathrm{C} 18^{\prime}$ & $1.345(5)$ \\
\hline $\mathrm{C} 2-\mathrm{C} 3$ & $1.419(3)$ & $\mathrm{C} 17-\mathrm{C} 18$ & $1.405(6)$ \\
\hline $\mathrm{C} 2-\mathrm{H} 2$ & 0.9500 & $\mathrm{C} 17-\mathrm{C} 17^{\mathrm{i}}$ & $1.487(4)$ \\
\hline $\mathrm{C} 3-\mathrm{C} 4$ & $1.356(3)$ & $\mathrm{C} 18-\mathrm{C} 19$ & $1.386(7)$ \\
\hline $\mathrm{C} 3-\mathrm{H} 3$ & 0.9500 & C18-H18 & 0.9500 \\
\hline $\mathrm{C} 4-\mathrm{C} 5$ & $1.448(3)$ & C19-H19 & 0.9500 \\
\hline $\mathrm{C} 6-\mathrm{C} 7$ & $1.447(3)$ & $\mathrm{C} 18^{\prime}-\mathrm{C} 19^{\prime}$ & $1.389(6)$ \\
\hline C6- $-\mathrm{H} 6$ & 0.9500 & $\mathrm{C} 18^{\prime}-\mathrm{H} 18^{\prime}$ & 0.9500 \\
\hline $\mathrm{C} 7-\mathrm{C} 12$ & $1.402(3)$ & $\mathrm{C} 19^{\prime}-\mathrm{H} 19^{\prime}$ & 0.9500 \\
\hline $\mathrm{O} 6-\mathrm{Mo1}-\mathrm{O} 5$ & $105.24(7)$ & $\mathrm{C} 8-\mathrm{C} 9-\mathrm{H} 9$ & 120.0 \\
\hline $\mathrm{O} 6-\mathrm{Mo1}-\mathrm{O} 3$ & $97.40(7)$ & $\mathrm{C} 10-\mathrm{C} 9-\mathrm{H} 9$ & 120.0 \\
\hline $\mathrm{O} 5-\mathrm{Mo} 1-\mathrm{O} 3$ & $103.06(7)$ & $\mathrm{C} 11-\mathrm{C} 10-\mathrm{C} 9$ & $120.2(2)$ \\
\hline $\mathrm{O} 6-\mathrm{Mo} 1-\mathrm{O} 2$ & $93.86(7)$ & $\mathrm{C} 11-\mathrm{C} 10-\mathrm{H} 10$ & 119.9 \\
\hline $\mathrm{O} 5-\mathrm{Mo} 1-\mathrm{O} 2$ & $98.70(7)$ & $\mathrm{C} 9-\mathrm{C} 10-\mathrm{H} 10$ & 119.9 \\
\hline $\mathrm{O} 3-\mathrm{Mo1}-\mathrm{O} 2$ & $151.84(6)$ & $\mathrm{O} 4-\mathrm{C} 11-\mathrm{C} 10$ & $125.6(2)$ \\
\hline $\mathrm{O} 6-\mathrm{Mo1}-\mathrm{O} 1 \mathrm{~W}$ & $170.71(7)$ & $\mathrm{O} 4-\mathrm{C} 11-\mathrm{C} 12$ & $114.2(2)$ \\
\hline $\mathrm{O} 5-\mathrm{Mo} 1-\mathrm{O} 1 \mathrm{~W}$ & $82.87(6)$ & $\mathrm{C} 10-\mathrm{C} 11-\mathrm{C} 12$ & $120.2(2)$ \\
\hline
\end{tabular}




\begin{tabular}{|c|c|c|c|}
\hline $\mathrm{O} 3-\mathrm{Mo1}-\mathrm{O} 1 \mathrm{~W}$ & $84.91(6)$ & $\mathrm{O} 3-\mathrm{C} 12-\mathrm{C} 7$ & $123.18(19)$ \\
\hline $\mathrm{O} 2-\mathrm{Mo} 1-\mathrm{O} 1 \mathrm{~W}$ & $80.25(6)$ & $\mathrm{O} 3-\mathrm{C} 12-\mathrm{C} 11$ & $117.5(2)$ \\
\hline $\mathrm{O} 6-\mathrm{Mo1}-\mathrm{N} 2$ & $95.98(7)$ & $\mathrm{C} 7-\mathrm{C} 12-\mathrm{C} 11$ & $119.3(2)$ \\
\hline $\mathrm{O} 5-\mathrm{Mo} 1-\mathrm{N} 2$ & $157.48(7)$ & $\mathrm{O} 4-\mathrm{C} 13-\mathrm{C} 14$ & $106.5(2)$ \\
\hline $\mathrm{O} 3-\mathrm{Mo} 1-\mathrm{N} 2$ & $81.20(6)$ & $\mathrm{O} 4-\mathrm{C} 13-\mathrm{H} 13 \mathrm{~A}$ & 110.4 \\
\hline $\mathrm{O} 2-\mathrm{Mo} 1-\mathrm{N} 2$ & $71.99(6)$ & $\mathrm{C} 14-\mathrm{C} 13-\mathrm{H} 13 \mathrm{~A}$ & 110.4 \\
\hline $\mathrm{O} 1 \mathrm{~W}-\mathrm{Mo} 1-\mathrm{N} 2$ & $75.43(6)$ & $\mathrm{O} 4-\mathrm{C} 13-\mathrm{H} 13 \mathrm{~B}$ & 110.4 \\
\hline $\mathrm{C} 4-\mathrm{O} 1-\mathrm{C} 1$ & $105.58(18)$ & $\mathrm{C} 14-\mathrm{C} 13-\mathrm{H} 13 \mathrm{~B}$ & 110.4 \\
\hline $\mathrm{C} 5-\mathrm{O} 2-\mathrm{Mo} 1$ & $118.96(13)$ & $\mathrm{H} 13 \mathrm{~A}-\mathrm{C} 13-\mathrm{H} 13 \mathrm{~B}$ & 108.6 \\
\hline $\mathrm{C} 12-\mathrm{O} 3-\mathrm{Mo} 1$ & $134.52(14)$ & $\mathrm{C} 13-\mathrm{C} 14-\mathrm{H} 14 \mathrm{~A}$ & 109.5 \\
\hline $\mathrm{C} 11-\mathrm{O} 4-\mathrm{C} 13$ & $118.0(2)$ & $\mathrm{C} 13-\mathrm{C} 14-\mathrm{H} 14 \mathrm{~B}$ & 109.5 \\
\hline Mo1-O1W-H11 & $120.4(18)$ & $\mathrm{H} 14 \mathrm{~A}-\mathrm{C} 14-\mathrm{H} 14 \mathrm{~B}$ & 109.5 \\
\hline Mo1-O1W-H12 & $128.8(19)$ & $\mathrm{C} 13-\mathrm{C} 14-\mathrm{H} 14 \mathrm{C}$ & 109.5 \\
\hline $\mathrm{H} 11-\mathrm{O} 1 \mathrm{~W}-\mathrm{H} 12$ & $110.1(17)$ & $\mathrm{H} 14 \mathrm{~A}-\mathrm{C} 14-\mathrm{H} 14 \mathrm{C}$ & 109.5 \\
\hline $\mathrm{C} 5-\mathrm{N} 1-\mathrm{N} 2$ & $109.13(17)$ & $\mathrm{H} 14 \mathrm{~B}-\mathrm{C} 14-\mathrm{H} 14 \mathrm{C}$ & 109.5 \\
\hline $\mathrm{C} 6-\mathrm{N} 2-\mathrm{N} 1$ & $115.97(17)$ & $\mathrm{N} 3-\mathrm{C} 15-\mathrm{C} 16$ & $124.9(4)$ \\
\hline $\mathrm{C} 6-\mathrm{N} 2-\mathrm{Mo} 1$ & $128.78(14)$ & N3-C15-H15 & 117.5 \\
\hline $\mathrm{N} 1-\mathrm{N} 2-\mathrm{Mo} 1$ & $115.24(12)$ & $\mathrm{C} 16-\mathrm{C} 15-\mathrm{H} 15$ & 117.5 \\
\hline $\mathrm{C} 15-\mathrm{N} 3-\mathrm{C} 19$ & $116.8(4)$ & $\mathrm{C} 17-\mathrm{C} 16-\mathrm{C} 15$ & $119.5(4)$ \\
\hline $\mathrm{C} 19^{\prime}-\mathrm{N} 3-\mathrm{C} 15^{\prime}$ & $117.2(3)$ & $\mathrm{C} 17-\mathrm{C} 16-\mathrm{H} 16$ & 120.2 \\
\hline $\mathrm{C} 2-\mathrm{C} 1-\mathrm{O} 1$ & $110.9(2)$ & $\mathrm{C} 15-\mathrm{C} 16-\mathrm{H} 16$ & 120.2 \\
\hline $\mathrm{C} 2-\mathrm{C} 1-\mathrm{H} 1$ & 124.5 & $\mathrm{C} 16^{\prime}-\mathrm{C} 15^{\prime}-\mathrm{N} 3$ & $120.4(4)$ \\
\hline $\mathrm{O} 1-\mathrm{C} 1-\mathrm{H} 1$ & 124.5 & $\mathrm{C} 16^{\prime}-\mathrm{C} 15^{\prime}-\mathrm{H} 15^{\prime}$ & 119.8 \\
\hline $\mathrm{C} 1-\mathrm{C} 2-\mathrm{C} 3$ & $106.6(2)$ & $\mathrm{N} 3-\mathrm{C} 15^{\prime}-\mathrm{H} 15^{\prime}$ & 119.8 \\
\hline $\mathrm{C} 1-\mathrm{C} 2-\mathrm{H} 2$ & 126.7 & $\mathrm{C} 15^{\prime}-\mathrm{C} 16^{\prime}-\mathrm{C} 17$ & $116.0(3)$ \\
\hline $\mathrm{C} 3-\mathrm{C} 2-\mathrm{H} 2$ & 126.7 & $\mathrm{C} 15^{\prime}-\mathrm{C} 16^{\prime}-\mathrm{H} 16^{\prime}$ & 122.0 \\
\hline $\mathrm{C} 4-\mathrm{C} 3-\mathrm{C} 2$ & $106.1(2)$ & $\mathrm{C} 17-\mathrm{C} 16^{\prime}-\mathrm{H} 16^{\prime}$ & 122.0 \\
\hline $\mathrm{C} 4-\mathrm{C} 3-\mathrm{H} 3$ & 126.9 & $\mathrm{C} 16-\mathrm{C} 17-\mathrm{C} 18$ & $115.8(3)$ \\
\hline $\mathrm{C} 2-\mathrm{C} 3-\mathrm{H} 3$ & 126.9 & $\mathrm{C} 18^{\prime}-\mathrm{C} 17-\mathrm{C} 17^{\mathrm{i}}$ & $122.4(3)$ \\
\hline $\mathrm{C} 3-\mathrm{C} 4-\mathrm{O} 1$ & $110.83(19)$ & $\mathrm{C} 16-\mathrm{C} 17-\mathrm{C} 17^{\mathrm{i}}$ & $122.2(3)$ \\
\hline $\mathrm{C} 3-\mathrm{C} 4-\mathrm{C} 5$ & $130.1(2)$ & $\mathrm{C} 18-\mathrm{C} 17-\mathrm{C} 17^{\mathrm{i}}$ & $120.9(3)$ \\
\hline $\mathrm{O} 1-\mathrm{C} 4-\mathrm{C} 5$ & $119.02(18)$ & $\mathrm{C} 18^{\prime}-\mathrm{C} 17-\mathrm{C} 16^{\prime}$ & $117.6(3)$ \\
\hline $\mathrm{N} 1-\mathrm{C} 5-\mathrm{O} 2$ & $124.54(19)$ & $\mathrm{C} 17^{\mathrm{i}}-\mathrm{C} 17-\mathrm{C} 16^{\prime}$ & $117.9(3)$ \\
\hline $\mathrm{N} 1-\mathrm{C} 5-\mathrm{C} 4$ & $119.62(19)$ & $\mathrm{C} 19-\mathrm{C} 18-\mathrm{C} 17$ & $119.3(5)$ \\
\hline $\mathrm{O} 2-\mathrm{C} 5-\mathrm{C} 4$ & $115.83(18)$ & $\mathrm{C} 19-\mathrm{C} 18-\mathrm{H} 18$ & 120.4 \\
\hline $\mathrm{N} 2-\mathrm{C} 6-\mathrm{C} 7$ & $123.35(19)$ & $\mathrm{C} 17-\mathrm{C} 18-\mathrm{H} 18$ & 120.4 \\
\hline $\mathrm{N} 2-\mathrm{C} 6-\mathrm{H} 6$ & 118.3 & N3-C19-C18 & $121.9(6)$ \\
\hline $\mathrm{C} 7-\mathrm{C} 6-\mathrm{H} 6$ & 118.3 & N3-C19-H19 & 119.1 \\
\hline $\mathrm{C} 12-\mathrm{C} 7-\mathrm{C} 8$ & $119.5(2)$ & $\mathrm{C} 18-\mathrm{C} 19-\mathrm{H} 19$ & 119.1 \\
\hline $\mathrm{C} 12-\mathrm{C} 7-\mathrm{C} 6$ & $122.4(2)$ & $\mathrm{C} 17-\mathrm{C} 18^{\prime}-\mathrm{C} 19^{\prime}$ & $120.1(4)$ \\
\hline $\mathrm{C} 8-\mathrm{C} 7-\mathrm{C} 6$ & $118.11(19)$ & $\mathrm{C} 17-\mathrm{C} 18^{\prime}-\mathrm{H} 18^{\prime}$ & 119.9 \\
\hline $\mathrm{C} 9-\mathrm{C} 8-\mathrm{C} 7$ & $120.7(2)$ & $\mathrm{C} 19^{\prime}-\mathrm{C} 18^{\prime}-\mathrm{H} 18^{\prime}$ & 119.9 \\
\hline $\mathrm{C} 9-\mathrm{C} 8-\mathrm{H} 8$ & 119.7 & $\mathrm{~N} 3-\mathrm{C} 19^{\prime}-\mathrm{C} 18^{\prime}$ & $124.6(4)$ \\
\hline $\mathrm{C} 7-\mathrm{C} 8-\mathrm{H} 8$ & 119.7 & $\mathrm{~N} 3-\mathrm{C} 19^{\prime}-\mathrm{H} 19^{\prime}$ & 117.7 \\
\hline $\mathrm{C} 8-\mathrm{C} 9-\mathrm{C} 10$ & $120.0(2)$ & $\mathrm{C} 18^{\prime}-\mathrm{C} 19^{\prime}-\mathrm{H} 19^{\prime}$ & 117.7 \\
\hline $\mathrm{O} 6-\mathrm{Mo} 1-\mathrm{O} 2-\mathrm{C} 5$ & $97.28(15)$ & $\mathrm{C} 13-\mathrm{O} 4-\mathrm{C} 11-\mathrm{C} 12$ & $179.4(2)$ \\
\hline $\mathrm{O} 5-\mathrm{Mo} 1-\mathrm{O} 2-\mathrm{C} 5$ & $-156.65(15)$ & $\mathrm{C} 9-\mathrm{C} 10-\mathrm{C} 11-\mathrm{O} 4$ & $177.9(2)$ \\
\hline
\end{tabular}




\begin{tabular}{|c|c|c|c|}
\hline $\mathrm{O} 3-\mathrm{Mo} 1-\mathrm{O} 2-\mathrm{C} 5$ & $-16.3(2)$ & $\mathrm{C} 9-\mathrm{C} 10-\mathrm{C} 11-\mathrm{C} 12$ & $-2.1(4)$ \\
\hline $\mathrm{O} 1 \mathrm{~W}-\mathrm{Mo} 1-\mathrm{O} 2-\mathrm{C} 5$ & $-75.49(14)$ & $\mathrm{Mo} 1-\mathrm{O} 3-\mathrm{C} 12-\mathrm{C} 7$ & $-32.0(3)$ \\
\hline $\mathrm{N} 2-\mathrm{Mo} 1-\mathrm{O} 2-\mathrm{C} 5$ & $2.25(14)$ & $\mathrm{Mo} 1-\mathrm{O} 3-\mathrm{C} 12-\mathrm{C} 11$ & $149.69(17)$ \\
\hline $\mathrm{O} 6-\mathrm{Mo1}-\mathrm{O} 3-\mathrm{C} 12$ & $-66.0(2)$ & $\mathrm{C} 8-\mathrm{C} 7-\mathrm{C} 12-\mathrm{O} 3$ & $-175.3(2)$ \\
\hline $\mathrm{O} 5-\mathrm{Mo} 1-\mathrm{O} 3-\mathrm{C} 12$ & $-173.64(19)$ & $\mathrm{C} 6-\mathrm{C} 7-\mathrm{C} 12-\mathrm{O} 3$ & $5.3(3)$ \\
\hline $\mathrm{O} 2-\mathrm{Mo} 1-\mathrm{O} 3-\mathrm{C} 12$ & $46.7(3)$ & $\mathrm{C} 8-\mathrm{C} 7-\mathrm{C} 12-\mathrm{C} 11$ & $3.0(3)$ \\
\hline $\mathrm{O} 1 \mathrm{~W}-\mathrm{Mo} 1-\mathrm{O} 3-\mathrm{C} 12$ & $104.91(19)$ & $\mathrm{C} 6-\mathrm{C} 7-\mathrm{C} 12-\mathrm{C} 11$ & $-176.4(2)$ \\
\hline $\mathrm{N} 2-\mathrm{Mo} 1-\mathrm{O} 3-\mathrm{C} 12$ & $28.90(19)$ & $\mathrm{O} 4-\mathrm{C} 11-\mathrm{C} 12-\mathrm{O} 3$ & $-2.0(3)$ \\
\hline $\mathrm{C} 5-\mathrm{N} 1-\mathrm{N} 2-\mathrm{C} 6$ & $-177.34(18)$ & $\mathrm{C} 10-\mathrm{C} 11-\mathrm{C} 12-\mathrm{O} 3$ & $178.0(2)$ \\
\hline $\mathrm{C} 5-\mathrm{N} 1-\mathrm{N} 2-\mathrm{Mo} 1$ & $3.9(2)$ & $\mathrm{O} 4-\mathrm{C} 11-\mathrm{C} 12-\mathrm{C} 7$ & $179.61(19)$ \\
\hline $\mathrm{O} 6-\mathrm{Mo} 1-\mathrm{N} 2-\mathrm{C} 6$ & $85.90(18)$ & $\mathrm{C} 10-\mathrm{C} 11-\mathrm{C} 12-\mathrm{C} 7$ & $-0.3(3)$ \\
\hline $\mathrm{O} 5-\mathrm{Mo} 1-\mathrm{N} 2-\mathrm{C} 6$ & $-113.6(2)$ & $\mathrm{C} 11-\mathrm{O} 4-\mathrm{C} 13-\mathrm{C} 14$ & $180.0(2)$ \\
\hline $\mathrm{O} 3-\mathrm{Mo} 1-\mathrm{N} 2-\mathrm{C} 6$ & $-10.70(18)$ & $\mathrm{C} 19^{\prime}-\mathrm{N} 3-\mathrm{C} 15-\mathrm{C} 16$ & $16.4(3)$ \\
\hline $\mathrm{O} 2-\mathrm{Mo} 1-\mathrm{N} 2-\mathrm{C} 6$ & $178.02(19)$ & $\mathrm{C} 19-\mathrm{N} 3-\mathrm{C} 15-\mathrm{C} 16$ & $4.2(3)$ \\
\hline $\mathrm{O} 1 \mathrm{~W}-\mathrm{Mo} 1-\mathrm{N} 2-\mathrm{C} 6$ & $-97.69(18)$ & $\mathrm{C} 15^{\prime}-\mathrm{N} 3-\mathrm{C} 15-\mathrm{C} 16$ & $-89.7(5)$ \\
\hline O6-Mo1-N2-N1 & $-95.50(14)$ & $\mathrm{N} 3-\mathrm{C} 15-\mathrm{C} 16-\mathrm{C} 17$ & $6.3(3)$ \\
\hline $\mathrm{O} 5-\mathrm{Mo} 1-\mathrm{N} 2-\mathrm{N} 1$ & $65.0(2)$ & $\mathrm{C} 15-\mathrm{N} 3-\mathrm{C} 15^{\prime}-\mathrm{C} 16^{\prime}$ & $76.3(5)$ \\
\hline $\mathrm{O} 3-\mathrm{Mo} 1-\mathrm{N} 2-\mathrm{N} 1$ & $167.90(14)$ & $\mathrm{C} 19^{\prime}-\mathrm{N} 3-\mathrm{C} 15^{\prime}-\mathrm{C} 16^{\prime}$ & $-12.7(5)$ \\
\hline $\mathrm{O} 2-\mathrm{Mo} 1-\mathrm{N} 2-\mathrm{N} 1$ & $-3.38(12)$ & $\mathrm{C} 19-\mathrm{N} 3-\mathrm{C} 15^{\prime}-\mathrm{C} 16^{\prime}$ & $-25.7(5)$ \\
\hline $\mathrm{O} 1 \mathrm{~W}-\mathrm{Mo} 1-\mathrm{N} 2-\mathrm{N} 1$ & $80.91(13)$ & $\mathrm{N} 3-\mathrm{C} 15^{\prime}-\mathrm{C} 16^{\prime}-\mathrm{C} 17$ & $-4.0(5)$ \\
\hline $\mathrm{C} 4-\mathrm{O} 1-\mathrm{C} 1-\mathrm{C} 2$ & $0.5(3)$ & $\mathrm{C} 15-\mathrm{C} 16-\mathrm{C} 17-\mathrm{C} 18^{\prime}$ & $-25.2(4)$ \\
\hline $\mathrm{O} 1-\mathrm{C} 1-\mathrm{C} 2-\mathrm{C} 3$ & $-0.4(3)$ & $\mathrm{C} 15-\mathrm{C} 16-\mathrm{C} 17-\mathrm{C} 18$ & $-14.9(4)$ \\
\hline $\mathrm{C} 1-\mathrm{C} 2-\mathrm{C} 3-\mathrm{C} 4$ & $0.1(3)$ & $\mathrm{C} 15-\mathrm{C} 16-\mathrm{C} 17-\mathrm{C} 17^{\mathrm{i}}$ & $177.4(3)$ \\
\hline $\mathrm{C} 2-\mathrm{C} 3-\mathrm{C} 4-\mathrm{O} 1$ & $0.2(3)$ & $\mathrm{C} 15-\mathrm{C} 16-\mathrm{C} 17-\mathrm{C} 16^{\prime}$ & $82.9(4)$ \\
\hline $\mathrm{C} 2-\mathrm{C} 3-\mathrm{C} 4-\mathrm{C} 5$ & $-179.5(2)$ & $\mathrm{C} 15^{\prime}-\mathrm{C} 16^{\prime}-\mathrm{C} 17-\mathrm{C} 18^{\prime}$ & $18.8(6)$ \\
\hline $\mathrm{C} 1-\mathrm{O} 1-\mathrm{C} 4-\mathrm{C} 3$ & $-0.4(2)$ & $\mathrm{C} 15^{\prime}-\mathrm{C} 16^{\prime}-\mathrm{C} 17-\mathrm{C} 16$ & $-70.2(4)$ \\
\hline $\mathrm{C} 1-\mathrm{O} 1-\mathrm{C} 4-\mathrm{C} 5$ & $179.28(19)$ & $\mathrm{C} 15^{\prime}-\mathrm{C} 16^{\prime}-\mathrm{C} 17-\mathrm{C} 18$ & $30.1(5)$ \\
\hline $\mathrm{N} 2-\mathrm{N} 1-\mathrm{C} 5-\mathrm{O} 2$ & $-2.2(3)$ & $\mathrm{C} 15^{\prime}-\mathrm{C} 16^{\prime}-\mathrm{C} 17-\mathrm{C} 17^{\mathrm{i}}$ & $-177.6(3)$ \\
\hline $\mathrm{N} 2-\mathrm{N} 1-\mathrm{C} 5-\mathrm{C} 4$ & $179.02(17)$ & $\mathrm{C} 18^{\prime}-\mathrm{C} 17-\mathrm{C} 18-\mathrm{C} 19$ & $80(2)$ \\
\hline $\mathrm{Mo} 1-\mathrm{O} 2-\mathrm{C} 5-\mathrm{N} 1$ & $-0.9(3)$ & $\mathrm{C} 16-\mathrm{C} 17-\mathrm{C} 18-\mathrm{C} 19$ & $13.7(6)$ \\
\hline $\mathrm{Mo} 1-\mathrm{O} 2-\mathrm{C} 5-\mathrm{C} 4$ & $177.96(13)$ & $\mathrm{C} 17^{\mathrm{i}}-\mathrm{C} 17-\mathrm{C} 18-\mathrm{C} 19$ & $-178.5(5)$ \\
\hline $\mathrm{C} 3-\mathrm{C} 4-\mathrm{C} 5-\mathrm{N} 1$ & $-179.8(2)$ & $\mathrm{C} 16^{\prime}-\mathrm{C} 17-\mathrm{C} 18-\mathrm{C} 19$ & $-27.0(6)$ \\
\hline $\mathrm{O} 1-\mathrm{C} 4-\mathrm{C} 5-\mathrm{N} 1$ & $0.6(3)$ & $\mathrm{C} 15-\mathrm{N} 3-\mathrm{C} 19-\mathrm{C} 18$ & $-5.3(6)$ \\
\hline $\mathrm{C} 3-\mathrm{C} 4-\mathrm{C} 5-\mathrm{O} 2$ & $1.3(3)$ & $\mathrm{C} 19^{\prime}-\mathrm{N} 3-\mathrm{C} 19-\mathrm{C} 18$ & $-76(2)$ \\
\hline $\mathrm{O} 1-\mathrm{C} 4-\mathrm{C} 5-\mathrm{O} 2$ & $-178.33(18)$ & $\mathrm{C} 15^{\prime}-\mathrm{N} 3-\mathrm{C} 19-\mathrm{C} 18$ & $30.5(6)$ \\
\hline $\mathrm{N} 1-\mathrm{N} 2-\mathrm{C} 6-\mathrm{C} 7$ & $177.65(18)$ & $\mathrm{C} 17-\mathrm{C} 18-\mathrm{C} 19-\mathrm{N} 3$ & $-3.8(7)$ \\
\hline $\mathrm{Mo} 1-\mathrm{N} 2-\mathrm{C} 6-\mathrm{C} 7$ & $-3.8(3)$ & $\mathrm{C} 16-\mathrm{C} 17-\mathrm{C} 18^{\prime}-\mathrm{C} 19^{\prime}$ & $22.4(7)$ \\
\hline $\mathrm{N} 2-\mathrm{C} 6-\mathrm{C} 7-\mathrm{C} 12$ & $10.2(3)$ & $\mathrm{C} 18-\mathrm{C} 17-\mathrm{C} 18^{\prime}-\mathrm{C} 19^{\prime}$ & $-95(2)$ \\
\hline $\mathrm{N} 2-\mathrm{C} 6-\mathrm{C} 7-\mathrm{C} 8$ & $-169.2(2)$ & $\mathrm{C} 17^{\mathrm{i}}-\mathrm{C} 17-\mathrm{C} 18^{\prime}-\mathrm{C} 19^{\prime}$ & $179.8(5)$ \\
\hline $\mathrm{C} 12-\mathrm{C} 7-\mathrm{C} 8-\mathrm{C} 9$ & $-3.2(3)$ & $\mathrm{C} 16^{\prime}-\mathrm{C} 17-\mathrm{C} 18^{\prime}-\mathrm{C} 19^{\prime}$ & $-17.4(7)$ \\
\hline $\mathrm{C} 6-\mathrm{C} 7-\mathrm{C} 8-\mathrm{C} 9$ & $176.2(2)$ & $\mathrm{C} 15-\mathrm{N} 3-\mathrm{C} 19^{\prime}-\mathrm{C} 18^{\prime}$ & $-19.5(7)$ \\
\hline $\mathrm{C} 7-\mathrm{C} 8-\mathrm{C} 9-\mathrm{C} 10$ & $0.8(4)$ & $\mathrm{C} 19-\mathrm{N} 3-\mathrm{C} 19^{\prime}-\mathrm{C} 18^{\prime}$ & $95(2)$ \\
\hline $\mathrm{C} 8-\mathrm{C} 9-\mathrm{C} 10-\mathrm{C} 11$ & $1.9(4)$ & $\mathrm{C} 15^{\prime}-\mathrm{N} 3-\mathrm{C} 19^{\prime}-\mathrm{C} 18^{\prime}$ & $15.7(7)$ \\
\hline $\mathrm{C} 13-\mathrm{O} 4-\mathrm{C} 11-\mathrm{C} 10$ & $-0.6(3)$ & $\mathrm{C} 17-\mathrm{C} 18^{\prime}-\mathrm{C} 19^{\prime}-\mathrm{N} 3$ & $-0.3(9)$ \\
\hline
\end{tabular}

Symmetry code: (i) $-x+3,-y,-z+1$. 
supporting information

Hydrogen-bond geometry $\left(\AA,{ }^{\circ}\right)$

\begin{tabular}{lllll}
\hline$D-\mathrm{H} \cdots A$ & $D-\mathrm{H}$ & $\mathrm{H} \cdots A$ & $D \cdots A$ & $D-\mathrm{H} \cdots A$ \\
\hline $\mathrm{O} 1 \mathrm{w}-\mathrm{H} 11 \cdots \mathrm{N} 3$ & $0.83(1)$ & $1.86(1)$ & $2.689(3)$ & $174(3)$ \\
$\mathrm{O} 1 \mathrm{w}-\mathrm{H} 12 \cdots \mathrm{N} 1^{1 i}$ & $0.84(1)$ & $1.97(1)$ & $2.794(2)$ & $167(3)$ \\
\hline
\end{tabular}

Symmetry code: (ii) $-x+2,-y+1,-z$. 